
\section{unpredictable environments \\ Offspring size variation within broods as a bet-hedging strategy in}

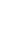

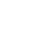

\author{
Dustin J. Marshall ${ }^{1}$, Russell Bonduriansky ${ }^{2}$ \& Luc F. Bussière Re,4 $^{2,3}$
}

${ }^{I}$ School of Integrative Biology/Centre for Marine Studies, The University of Queensland, 4072, Qld, Australia;

${ }^{2}$ Evolution \& Ecology Research Centre and School of Biological, Earth \& Environmental Sciences, University of New South Wales, 2052, NSW, Australia;

$1{ }^{3}$ Zoologisches Museum der Universität Zürich, Winterthurerstrasse 190, CH 8057, Zürich, Switzerland

${ }^{4}$ Current address: School of Biological and Environmental Sciences, University of Stirling, FK9 4LA, Stirling,

3 United Kingdom

4

\section{d.marshall1@uq.edu.au}

r.bonduriansky@unsw.edu.au

luc.bussiere@stir.ac.uk

20 Keywords: egg size, maternal effect, optimality, seed size, life history, reproductive strategy, environmental

variation, unpredictable environments

2

3


24 Offspring size is strikingly variable within species. Although theory can account for variation

25 in offspring size among mothers, an adaptive explanation for variation within individual

26 broods has proven elusive. Theoretical considerations of this problem assume that producing

27 offspring that are too small results in reduced offspring viability, but producing offspring that

28 are too large (for that environment) results only in a lost opportunity for increased fecundity.

29 However, logic and recent evidence suggest that offspring above a certain size will also have

30 lower fitness, such that mothers face fitness penalties on either side of an optimum. Although

31 theory assuming intermediate optima has been developed for other diversification traits, the

32 implications of this idea for selection on intra-brood variance in offspring size have not been

33 explored theoretically. Here we model the fitness of mothers producing offspring of uniform

34 vs. variable size in unpredictably variable environments and compare these two strategies

35 under a variety of conditions. Our model predicts that producing variably sized offspring

36 results in higher mean maternal fitness and less variation in fitness among generations when

37 there is a maximum and minimum viable offspring size, and many mothers under- or over-

38 estimate this optimum. This effect is especially strong when the viable offspring size range is

39 narrow relative to the range of environmental variation. To determine whether this prediction

40 is consistent with empirical evidence, we compare within- and among-mother variation in

41 offspring size for 5 phyla of marine invertebrates with different developmental modes

42 corresponding to contrasting levels of environmental predictability. Our comparative analysis

43 reveals that in the developmental mode in which mothers are unlikely to anticipate the

44 relationship between offspring size and performance, size-variation within mothers exceeds

45 variation among mothers, but the converse is true when optimal offspring size is likely to be 
46 more predictable. Together, our results support the hypothesis that variation in offspring size

47 within broods can reflect an adaptive strategy for dealing with unpredictably variable

48 environments. We suggest that when there is a minimum and a maximum viable offspring

49 size and the environment is unpredictable, selection will act on both the mean and variance

50 of offspring size.

51

52 
For over 50 years, biologists have sought to understand the remarkable variation in

54 offspring size among species (Lack 1947, Bagenal, 1969) and the study of offspring size has

55 become an important branch of life-history research (Stearns 1992). The resources available

56 to mothers are finite, so they can produce either many small or fewer, large offspring. Larger

57 offspring typically have higher fitness than smaller offspring, thereby offsetting any decrease

58 in fecundity. Smith and Fretwell (1974) produced one of the first theoretical examinations of

59 how mothers optimally balance the size and number of offspring they produce. Their classic

60 study has formed the basis for most theory on offspring size/number trade-offs, and most

61 models published since share a number of features with their original work. These models

62 typically derive the best maternal allocation strategy assuming a trade-off between offspring

63 size and number and a positive correlation between offspring fitness and offspring size (e.g.

64 Vance 1973, Smith and Fretwell 1974, Sargent et al. 1987). The general prediction from this

65 work is that under constant environmental conditions (and thus a constant offspring size-

66 fitness relationship), a single offspring size will be optimal. However in nature, offspring

67 sizes are extremely variable within populations and offspring size can be correlated with a

68 number of maternal factors such as body size or nutrition (Turner and Lawrence 1977).

69 While the earliest models could not account for such variation, more recent models

70 incorporating the effects of maternal phenotype on the natal environment successfully predict

71 the observed variation in offspring sizes among different mothers (e.g. Parker and Begon

72 1986, Hendry et al. 2001, Sakai and Harada 2001). Empirical evidence supports these

73 models. For example, in species of fish where the maternal phenotype can affect the

74 offspring size-fitness relationship, offspring size variation within populations is relatively 
75 high (Einum and Fleming 2002). Similarly, Fox et al. (1997) showed that when mothers can

76 detect the likely environment of their offspring, they adjust the size of their offspring

77 accordingly suggesting that inter-individual variation in offspring size represents an adaptive

78 maternal effect.

79 Although theory can now account for variation in offspring size among mothers,

80 explaining variation in the size of offspring from the same mother remains problematic.

81 Offspring size shows remarkable variation within individual broods in many taxa (Turner and

82 Lawrence 1977, Williams 1994, Fox and Czesak, 2000, Kudo 2001, Marshall et al. 2003).

83 There are numerous verbal arguments for producing offspring that vary in size (e.g. Capinera

84 1979, Crump 1981, Lips 2001). In such considerations, within-brood variation is viewed as a

85 form of bet-hedging in which females ensure that at least a few offspring approach the

86 optimum in some unpredictable future environment (Koops et al. 2003). However, the few

87 formal models of within brood offspring size variation do not support such an adaptive

88 explanation for this variation (McGinley et al. 1987, Einum and Fleming 2004b, but see

89 Geritz [1995] for a rare exception using a game theoretic approach). In most instances,

90 producing a single offspring size within each brood is predicted to be optimal (note that

91 Vance [1973] and similar models predict two optima but one of these is simply a product of

92 infinitely small offspring retaining some fitness - an unrealistic situation). In the few cases

93 where producing variably-sized offspring within broods is favored in these models, it is

94 under restrictive and improbable conditions. For example, McGinley et al. (1987) found that

95 producing offspring of variable size was only advantageous when mothers could strictly

96 control the dispersal of their offspring. Similarly, Einum and Fleming (2004) found that

97 within-brood offspring size variation (described as diversified bet-hedging) was a less 
98 effective strategy than producing very large offspring (described as conservative bet-

99 hedging) for coping with environmental uncertainty. Consequently, within-brood variation in

100 offspring size is increasingly viewed as a product of physiological constraints that prevent

101 mothers from producing offspring of identical size, rather than as an adaptive strategy (Fox

102 and Czesak 2000, Einum and Fleming 2004b). In their review of offspring size effects in

103 insects, Fox and Czesak (2000, p. 358) concluded that ' $\ldots$...some authors have suggested that

104 at least some of the variation within families is an adaptive response to living in a variable

105 environment. At this time however, there are few experimental studies and too little

106 theoretical work to generalize." Thus, despite the intuitive appeal of intra-clutch variation in

107 offspring size as a mechanism for coping with environmental heterogeneity, theoretical

108 support for the concept remains elusive.

109 While offspring size theory has struggled to account for within-brood variation in

110 offspring size, parallel developments in the more general theory of bet-hedging have long

111 predicted a selection advantage for producing offspring with variable phenotypes. Cohen's

112 (1966) classic model and others since have shown that when the environment varies

113 unpredictably, mothers should produce offspring with a range of phenotypes either in a single

114 reproductive bout (Gillespie 1977, Bull 1987, Simons and Johnston 2006) or across multiple

115 reproductive bouts (Cooper and Kaplan 1982). Thus we face the puzzling situation where

116 general theory predicts a selection advantage for variation in offspring traits but specific

117 theory for offspring size typically does not.

118 The nature of optimality models may be partly responsible for the prevalence of

119 theory showing that within-brood offspring size variation is not adaptive. Most optimality

120 models use highly asymmetrical offspring fitness functions whereby, as offspring size 
121 increases, offspring fitness increases with diminishing returns, or levels off at a constant

122 maximum value (Smith and Fretwell 1974, McGinley et al. 1987, Einum and Fleming 2000,

123 2004b). In contrast, more general models of bet-hedging assume a symmetrical, curvilinear

124 relationship between offspring phenotype and fitness such that there are fitness penalties at

125 each end of the offspring phenotype continuum (e.g. Cohen 1966, Cooper and Kaplan 1982).

126 We suggest that offspring size models using an asymmetrical function are unrealistic and

127 underestimate the benefits of a diversified bet-hedging strategy in unpredictable

128 environments while overestimating the benefits of a conservative bet-hedging strategy (i.e.

129 producing offspring of a constant, large size).

130 The use of a Smith-Fretwell fitness function is problematic because it assumes that

131 above a certain size, larger offspring (including infinitely large offspring) have equal fitness.

132 Therefore, the only fitness cost to mothers of producing large offspring is a reduction in

133 fecundity. In nature, however, offspring exceeding a certain size will have lower fitness due

134 to physiological or anatomical constraints (Strathmann and Chaffee 1984, Congdon and

135 Gibbons 1987, Kaplan 1992, Strathmann 1995, Bernardo 1996), increased predation risk

136 (Dibattista et al. 2007) or an increased risk of polyspermy (Styan 1998, Marshall et al. 2002).

137 Thus offspring are likely to suffer a direct fitness cost if they are too small or too large for

138 their environment. If the environment varies unpredictably, then a conservative bet-hedging

139 strategy (i.e. simply producing larger offspring) is unlikely to insulate mothers from this

140 unpredictability because excessively large offspring will suffer reduced fitness. Furthermore,

141 with direct fitness costs on both sides of an optimum, the benefits of producing variably sized

142 offspring may be increased in unpredictable conditions. The benefits of within brood 
143 offspring size variation have not been modeled under the assumption that both very small and 144 very large offspring suffer reduced fitness.

145 Here, we compare the fitness of mothers employing contrasting reproductive

146 strategies: (1) an 'invariant' strategy where all offspring (or eggs) within a brood are of equal

147 size, and (2) a 'variable' strategy where offspring within broods vary in size. Like previous

148 authors, we found that this problem precludes a tractable analytic solution, and opted for a

149 simulation approach (Einum and Fleming 2004a). However, our approach differs from

150 previous studies in that we use a fitness function that penalizes offspring that are too large as

151 well as those that are too small for that environment. Recent empirical work has shown that

152 optimal offspring sizes can vary 2-fold over very small spatial scales within similar habitats

153 (Marshall et al. 2006, Marshall and Keough 2006), so the chances of mothers producing the

154 'wrong' offspring size for any particular environment are probably high. Therefore, we

155 varied the probability that mothers will produce offspring of a size that is not optimal for that

156 environment (i.e., make "errors"), and examined the relative fitness of the variable and

157 invariant reproductive strategies. Our simulation analysis suggested that, when mothers are

158 likely to make large errors with regard to optimal offspring size and viable offspring sizes are

159 bounded by a minimum and a maximum, within brood variation in offspring size increases

160 maternal fitness. We then examined whether species that were less able to predict the natal

161 environment produced more variably sized broods through a comparative analysis of

162 offspring size variation among marine invertebrate species that differ in their capacity to

163 predict the environment of their offspring (i.e. direct developers without dispersive young

164 versus indirect developers with highly dispersive young). A direct test of the predictions of

165 our model requires verifying the presumed causal link between a maximum viable egg size 
166 and selection on the variable strategy - a challenging problem. Our comparative analysis

167 does not constitute such a direct test. Nonetheless, the comparative analysis enables us to

168 determine whether the empirical evidence is consistent with our model's predictions.

\section{Materials and Methods}

\section{Model}

172 Following traditional offspring size models (e.g. Smith and Fretwell 1974), we

173 assume that there is a function linking egg fitness to egg size $(m)$, a minimum viable egg size

$174\left(m_{\min }\right)$, and a trade-off between the mean size of the eggs that a female produces and the

175 number of eggs that she can produce (Smith and Fretwell 1974). However, unlike previous

176 authors, we also assume that egg fitness decreases when egg size exceeds an optimum, so

177 that there is a maximum viable egg size $\left(m_{\max }\right)$. Thus, the fitness of an individual egg, $\phi(m)$,

178 is zero when egg size is less than $m_{\min }$ or greater than $m_{\max }$. In the viable range between $m_{\min }$ 179 and $m_{\max }$, egg fitness is given by

$$
\phi(m)=\frac{\left(1-\frac{m_{\min }}{m}\right)\left(1-\frac{m}{m_{\max }}\right)}{m},
$$

183 where $m$ is the size of an individual egg. This represents a convex function relating egg

184 fitness to egg size (see Appendix A: Fig. 1).

We assume that all females have an equal quantity of resources, $R$, to invest in

186 reproduction, and that there is a trade-off between the mean size of a female's eggs (brood 
187 mean, $\mu$ ), and the number of eggs that she can produce. Thus, the number of eggs, $N(\mu)$,

188 produced by a female with a brood-mean egg size $\mu$ is

$$
N(\mu)=R\left(\frac{M}{\mu}\right),
$$

191 where $M$ is the mean egg size in the population (equal to the optimum egg size and the 192 expected value of the distribution of $\mu$; see below). Consequently, females whose mean egg

193 size $(\mu)$ is smaller than the population average (i.e., $\mu<M$ ) produce more eggs than the 194 population average egg number (i.e., $N(\mu)>N(M)$ ), whereas females whose mean egg size is 195 greater than the population average (i.e., $\mu>M$ ) produce fewer eggs than the population 196 average (i.e., $N(\mu)<N(M)$ ), such that total reproductive output (the sum of the sizes of all 197 eggs produced by a female) is equal for all females.

198 The fitness of a particular mother, $W$, is thus equal to the summed fitnesses of all her 199 eggs,

$$
W=\sum^{N(\mu)} \phi(m),
$$

203 where $m$ is the size of a particular egg produced by a female with mean egg size $\mu$.

204 To determine whether the variable strategy could yield higher fitness than the 205 invariant strategy in unpredictably variable environments, we simulated different degrees of 206 environmental variability, and examined their consequences for the relative fitnesses (i.e., 207 recruitment rates) of two populations of 500 females, one population pursuing an invariant 208 reproductive strategy (no variation in egg size within broods), and the other population 
209 pursuing a variable reproductive strategy (within-brood variation in egg size). We used

210 populations, rather than a single individual, to represent each strategy within each generation

211 because this approach seems more biologically relevant, corresponding, for example, to a

212 situation where clonal populations of marine invertebrates or plants pursue contrasting

213 reproductive strategies. Simulating populations also greatly diminishes the probability of

214 genotype extinction, enabling us to analyse variation in strategy fitness within and across

215 generations as a continuous variable. Environmental variability $\left(\mathrm{CV}_{\mathrm{E}}\right)$ was a proxy for

216 mothers' abilities to predict the optimum brood-mean egg size: on average, the magnitude of

217 maternal "errors" (i.e., deviations from the optimum) increased with increasing

218 environmental variability. Note that we manipulated mean egg size for a population of

219 females rather than manipulating environmental parameters. This approach is functionally

220 equivalent to modeling environmental variation because the distribution of mismatches

221 between maternal mean egg size and environment are the same in each case. Our approach

222 has the advantage of allowing manipulation of variation in the mean distance from an

223 optimum independently of changes in minimum and maximum viable egg size. In each

224 simulation (representing a generation or reproductive bout), a brood-mean egg size, $\mu$, was

225 generated randomly for each of 500 mothers. The distribution of $\mu$ was log-normal, and its

226 expected value, $M$, always corresponded to the optimum egg size (i.e. the egg size that

227 maximizes maternal fitness). This assumes that the population mean is centered at the fitness

228 optimum, but that individuals within the population may over- or underestimate the optimum

229 egg size. The coefficient of variation of this distribution, $\mathrm{CV}_{\mathrm{E}}$, represented environmental

230 variability. We examined six degrees of environmental variability: $\mathrm{CV}_{\mathrm{E}}=0.3,0.6,1.0,1.15$,

231 1.3, 2.1. The biological significance of these values of $\mathrm{CV}_{\mathrm{E}}$ can be appreciated by inspecting 
232 their consequences for reproductive failure rate and among-generation variation in fitness

233 (see Results and Appendix A), which can be compared directly with parameters measured in

234 real populations. Indeed empirical studies strongly suggest that reproductive failure rates due

235 to producing offspring of the 'wrong' size can be even higher than those generated in our

236 simulations (Marshall and Keough 2007).

237 For each of the 500 brood-mean egg sizes, $\mu$, we then randomly generated $N(\mu)$

238 individual egg sizes, $m$, distributed normally about $\mu$ with standard deviation $\sigma$. Although

239 some eggs could thus have negative sizes, we assume that egg size is measured on an

240 arbitrary scale, and negative sizes simply yield zero fitness because they are less than $m_{\min }$.

241 For a given female with brood-mean egg size $\mu$, maternal fitness was then calculated in two

242 ways. First, maternal fitness under the invariant strategy, $W_{i n v}$, was calculated based on $N(\mu)$

243 eggs of size $\mu$. Second, maternal fitness under the variable strategy, $W_{v a r}$, was calculated

244 based on $N(\mu)$ eggs of different sizes, $m$. The arithmetic mean fitness of each strategy within

245 each generation (or reproductive bout) was then calculated from the fitnesses of the 500

246 females, and the relative fitness of the variable strategy ('Fitness differential', $\Delta W$ ) within a

247 generation was calculated as

$$
\Delta W=\frac{\left(\sum W_{v a r}-\sum W_{i n v}\right)}{\sum W_{i n v}},
$$

251 where $\sum W_{v a r}$ and $\sum W_{i n v}$ are the summed fitnesses of the 500 females based on the variable

252 and invariant strategies. Thus, $\Delta W<0$ indicates higher fitness for the invariant strategy,

253 whereas $\Delta W>0$ indicates higher fitness for the variable strategy within a generation or

254 reproductive bout. 
To examine how the magnitude of within brood variance in egg size affects maternal

256 fitness, we simulated different coefficients of within brood variation, $\mathrm{CV}_{\mathrm{B}}($ defined as $\sigma / \mu)$.

257 We examined values of $\mathrm{CV}_{\mathrm{B}}$ ranging from 0.01 to 0.5 because these values encompass those

258 observed in nature (Marshall and Keough 2007, Kohn and Perron, 1994). The value of $\sigma$ was

259 adjusted for each female so as to maintain a constant $\mathrm{CV}_{\mathrm{B}}$ despite variation in $\mu$.

260 To investigate how the shape of the function relating egg fitness to egg size affects

261 the performance of the variable strategy, we simulated every combination of $\mathrm{CV}_{\mathrm{E}}$ and $\mathrm{CV}_{\mathrm{B}}$

262 for three different egg fitness functions varying 16-fold in the width of the viable egg size

263 range (see Fig. 2). For each parameter combination, we conducted 500 simulations, with

264 different random distributions of $\mu$ and $m$ generated in each simulation, and tested the null

265 hypothesis $\Delta W=0$ by $t$-test. Where $\Delta W$ was not significantly different from zero, we

266 conducted up to 2000 additional simulations. Appendix A: Figure 1 illustrates the

267 performance of females under the variable and invariant strategy for several parameter

268 values. We checked our model for systematic bias by setting $\mathrm{CV}_{\mathrm{B}}$ to very small values, and

269 confirming that $\Delta W$ approaches zero as $\mathrm{CV}_{\mathrm{B}}$ approaches zero.

270 As an additional index of relative performance, we compared the coefficients of

271 variation of $\sum W_{v a r}$ and $\sum W_{i n v}$ over multiple simulations, assuming that the strategy exhibiting

272 less variation in mean fitness across generations enjoys a long-term advantage (Roff, 1992).

273 Simulations were conducted in Mathcad Plus 6.0 Professional Edition (C) MathSoft, Inc.,

274 Cambridge, Mass., U.S.A.).

275

276

Comparative Analysis 
Marine invertebrates are an ideal group to examine offspring size variation across

278 species because they are taxonomically diverse with a wide range of offspring dispersal

279 modes. Many marine invertebrate lineages exhibit a range of developmental modes,

280 indicating repeated, independent evolution of different developmental modes (Hart et al.

2812003 ) and multiple dispersal modes are present within some populations of the same species

282 (Krug 1998). Importantly, offspring size consistently has fitness consequences in marine

283 invertebrates and can be important at each life-history stage, sometimes in a conflicting

284 manner (Hart 1995, Levitan 1996, Moran and Emlet 2001, Marshall and Keough 2003,

285 Marshall et al. 2003).

286 Marine invertebrates can be divided into distinct developmental groups: direct

287 development, indirect development with no feeding, and indirect development with feeding.

288 Direct developers (D) typically produce relatively large offspring that either emerge from the

289 adult as a fully developed juvenile or emerge from an egg capsule as a juvenile. There is

290 extremely little dispersal during development and fertilisation in usually internal. Non-

291 feeding, indirect developers (NF) typically produce 'medium 'sized offspring that are

292 released from the adult as eggs (in the case of external fertilisers) or larvae (in the case of

293 internal fertilisers or brooders). The larvae typically have no feeding structures, relying on

294 maternal provisioning, and spend minutes to a few days in the plankton before encountering

295 a suitable habitat whereupon they metamorphose and begin adult life (Havenhand 1995).

296 Feeding, indirect developers (F) produce small eggs that are either externally or internally

297 fertilised and spend days to months feeding in the plankton. They may go through several

298 larval stages while in the plankton before metamorphosing and becoming an adult. Thus 
299 there is a clear ranking in the dispersal capabilities of D, NF and F offspring with D being the

300 least dispersive and $\mathrm{F}$ being the most dispersive.

301 There are clear differences in the ability of mothers with directly and indirectly

302 developing offspring to predict the relationship between offspring size and overall

303 performance (and thus the optimal offspring size that should be produced). Because

304 indirectly developing offspring are far more dispersive and can pass through multiple life-

305 history stages: i) the natal habitat (defined as the habitat in which offspring become

306 independent from maternal nutrition sources and begin to feed) is unlikely to be assessed by

307 mothers, ii) the natal habitat is unlikely to be affected by maternal phenotype and iii) there

308 can be conflicting selection pressures on offspring size among stages (Marshall et al. 2002).

309 These three factors all suggest that mothers with F offspring have a lower chance of

310 optimally provisioning individual offspring. In contrast, mothers with $\mathrm{D}$ offspring should be

311 better able to predict their offspring's environment. Because directly developing offspring are

312 released into the maternal environment there is at least the potential for mothers to assess the

313 environmental conditions and adaptively adjust the size of their offspring (e.g. Fox et al.

314 1997, Einum and Fleming 2002). Moreover, because direct developing offspring are

315 relatively weak dispersers, sibling competition and maternal phenotype are more likely to

316 affect the quality of the natal environment (i.e., essentially make it more predictable, Parker

317 and Begon 1986, Hendry et al. 2001). Finally, there is less potential for conflicting selection

318 pressures on offspring size in direct developers because they have fewer life-history stages

319 making the relationship between offspring size and performance more likely to be

320 predictable. If environmental unpredictability favours the production of variably sized

321 offspring, then we should see higher levels of variation within broods of $\mathrm{F}$ species than $\mathrm{D}$ 
322 species. Similarly, if the offspring environment of D species is more predictable, we should

323 see more variation among mothers in D species than F species. It is important to note that for

324 all the developmental groups, we expect there to be a minimum and maximum offspring size

325 that will be viable although the underlying causes of the upper constraints will probably

326 differ among the developmental modes. For example, physiological constraints probably

327 limit the maximum offspring size in direct developers but polyspermy effects limit offspring

328 size in broadcast spawners (Marshall and Keough 2007). Thus, while our theoretical analysis

329 considered the effects of environmental predictability on fitness under the assumption that

330 offspring can suffer fitness costs if they exceed an optimum size, our comparative analysis

331 examined the relationship between environmental predictability and components of variation

332 in offspring size in order to test our model's predictions. To determine whether the empirical

333 evidence was consistent with the predictions arising from our simulations, we compiled data

334 on variation in offspring size among and within marine invertebrate mothers from the

335 available literature and from unpublished data of our own (see Table 1). For more detailed

336 methods on our comparative analysis, see Appendix A.

Results

\section{Model}

340 Our simulations suggest that, in unpredictably variable environments, mothers

341 producing offspring of variable size within each brood (variable strategy) have higher mean

342 fitness within generations, as well as lower variance in fitness across generations, than

343 mothers producing offspring of uniform size (invariant strategy). This is because the 
344 production of offspring of different sizes insures that, for some females whose brood-mean

345 egg size falls outside the viable egg size range, some offspring are nonetheless able to 346 survive.

347 Within generations, the invariant strategy yields higher mean fitness when a large 348 proportion of females are able to produce mean egg sizes near the optimum, since all the 349 eggs produced by invariant strategy females have high fitness. However, when many females 350 produce mean egg sizes that deviate substantially from the optimum, the variable strategy 351 tends to do better on average because fewer females suffer total reproductive failure (see 352 below), and many females produce some eggs of near-optimum size, even if their brood353 mean egg size deviates from the optimum. When environmental variability is moderate (e.g., $\left.354 \mathrm{CV}_{\mathrm{E}}=0.6\right)$, a small degree of within brood variability in egg size (e.g., $\left.\mathrm{CV}_{\mathrm{B}}=0.01\right)$

355 nonetheless results in significantly higher mean maternal fitness than an invariant strategy, 356 although higher levels of within-brood variability are disadvantageous (Fig. 1; Appendix B:

357 Table 1). For example, egg fitness function ' $c$ ' in Fig. 1 yields a significant advantage for the 358 variable strategy (with $\mathrm{CV}_{\mathrm{B}}=0.01$ ) at $\mathrm{CV}_{\mathrm{E}}=0.6$, which corresponds to a reproductive failure 359 rate (i.e., probability of having no surviving offspring) of $24 \%$ for invariant strategy females. 360 When environmental variability is high (e.g., $\mathrm{CV}_{\mathrm{E}} \geq 1.3$, resulting in reproductive failure for $361>50 \%$ of invariant strategy females), maternal fitness increases with the degree of within362 brood variability $\left(\mathrm{CV}_{\mathrm{B}}\right)$. At intermediate levels of environmental variation (e.g., $\mathrm{CV}_{\mathrm{E}}=1.0$ to 363 1.15), a complex transition occurs where selection on $\mathrm{CV}_{\mathrm{B}}$ appears to be disruptive.

364 We investigated how the strength of stabilizing selection on egg size affects the 365 relative advantage of the variable strategy by examining three egg fitness functions differing 366 16-fold in the viable egg size range. The narrowest egg fitness function (function 'a' in Fig. 
367 1), representing the strongest stabilizing selection on egg size, results in the greatest

368 advantage for the variable strategy in variable environments (Appendix C: Fig. 2). However,

369 the difference between the two wider egg fitness functions (functions ' $b$ ' and ' $c$ ' in Fig. 1) is

370 less pronounced. This is because, given the trade-off between egg size and number, females

371 with large brood-mean egg sizes have few offspring and, thus, little influence on mean

372 fitness.

373 In addition, the variable strategy always results in lower variation in fitness across

374 generations (Fig. 2; Appendix B: Table 2), and this represents an additional advantage of the

375 variable strategy (see Roff 1992 and Discussion).

376 The fitness advantages of the variable reproductive strategy, both in terms of higher

377 mean fitness within generations and lower coefficients of variation in fitness across

378 generations, partly reflects lower rates of complete reproductive failure (Appendix C: Fig. 3).

379 For females pursuing the variable egg size strategy, reproductive failure rate declines with 380 increasing within-brood variability $\left(\mathrm{CV}_{\mathrm{B}}\right)($ Appendix $\mathrm{B}$ : Table 3$)$ and, even with the smallest

381 degree of within-brood variability $\left(\mathrm{CV}_{\mathrm{B}}=0.01\right)$, the variable strategy yields a significantly

382 lower reproductive failure rate than the invariant strategy (Sign test: $N=12500$ simulation

383 runs, $Z=111.66, P<0.0001)$. Reduced rates of reproductive failure thus moderate the effects

384 of environmental unpredictability.

385

\section{Comparative analysis}

The comparative data are consistent with the hypothesis that within-brood offspring

388 size variation reflects an adaptive maternal bet-hedging strategy in unpredictable

389 environments. The principle source of variation in offspring size strongly depends on the 
390 development type of that species (Table 2). For direct developers (where mothers have the

391 greatest ability to predict offspring environment), most of the variation in offspring size is at

392 the among-mother level (Fig. 3) whereas for indirect developers with feeding larvae (where

393 mothers have little ability to predict offspring environment), most of the variation is at the

394 within-mother level (Fig. 3). The indirect developers with non-feeding larvae are

395 intermediate to the two other groups, with equal levels of variation within and among

396 mothers. This pattern is maintained regardless of the taxonomic level at which it is tested

397 (Scale of variation $\times$ Development type interaction, family level: $F_{2,18}=7.05, P=0.0055$;

398 order level: $F_{2,15}=6.11, P=0.0011$; class level: $F_{2,8}=6.11, P=0.0245$; Fig. 3). Examining

399 within-mother variation in offspring size alone, there is a significant difference among

400 development types $\left(\mathrm{F}_{2,22}=5.54, \mathrm{P}=0.011\right)$ and within-mother variation is highest in indirect

401 developers with feeding larvae (Dunnett's test: F vs. NF: $\mathrm{P}<0.001$; F vs. D: $\mathrm{P}=0.04$ ).

\section{Discussion}

We found that when environments are unpredictable (such that mothers are likely to produce offspring of a mean size that deviates from the optimal size) and offspring fitness is maximized at an intermediate size, mothers producing a range of offspring sizes within a

407 brood (variable strategy) are likely to have higher fitness than mothers producing offspring of

408 identical size within broods (invariant strategy). Typically, the benefit of producing variably

409 sized offspring is assumed to be a reduction in among-generation variance in fitness (Seger

410 and Brockman 1987, Simons and Johnston 1997, Lips 2001, Laaksonen 2004), and our

411 analysis supports this view. This represents an advantage for the variable strategy because 
412 genotypes pursuing such a strategy will face a lower risk of extinction resulting from low

413 population size in some generations, and will have a higher net growth rate over multiple

414 generations (i.e. they will have a higher geometric mean fitness: for a detailed description of

415 why geometric mean fitness may be more important for selection, see Roff 1992 and Orr

416 2007). This reduction in among-generation variation in fitness (yielding a higher geometric

417 mean) forms the basis of bet-hedging theory: bet-hedgers don't necessarily do best all the

418 time, but they perform most consistently and are therefore favored by selection (Cohen 1966,

419 Roff 1992). However, our simulations show that mean fitness within generations is also

420 higher for mothers that produce offspring of variable size when the environment is

421 sufficiently variable (i.e. a higher arithmetic mean fitness) - a novel finding. Together, these

422 findings represent a large potential fitness advantage for the variable strategy in some

423 environments.

424 The finding that a variable strategy can attain higher fitness within generations

425 reflects the fact that the variable strategy outperforms the invariant strategy when brood-

426 mean egg size is displaced from the egg size optimum. Thus, the net relative fitness of the

427 variable strategy within generations reflects the average displacement of brood-mean egg

428 size from the egg size optimum, and a net advantage for the variable strategy can arise if the

429 mode of the distribution of brood-mean egg sizes is displaced from the egg size optimum. In

430 our simulations, the expected value of the brood-mean egg size distribution always coincides

431 with the optimum egg size, but the mode of the distribution is to the left of the egg size

432 optimum (as a consequence of the shape of the log-normal distribution), yielding a net

433 advantage within generations for the variable strategy under some parameter combinations.

434 We suggest that, in natural populations, the mode of the distribution of offspring size may 
435 often be shifted to the left of the optimum as a result of resource limitation, or classic bet-

436 hedging whereby genotypes that produce a greater number of small offspring may

437 outperform those that produce fewer, larger offspring when optimum egg size is difficult to

438 predict.

439 Our findings provide some of the first compelling theoretical support for the idea that

440 the production of heterogeneous broods, so often observed in nature, can reflect an adaptive

441 reproductive strategy that yields higher fitness within generations (see Kudo [2001] for an

442 interesting empirical example). Our results suggest that whenever there is a minimum and a

443 maximum viable offspring size, there are two ecological conditions under which producing

444 offspring of variable size may be favored: highly unpredictable environments and narrow

445 fitness functions. There is good evidence suggesting that optimal offspring sizes can vary

446 considerably, even over small spatial and temporal scales due to a range of physical (e.g.

447 desiccation stress on either side of a surge channel; Moran and Emlet 2001) and biological

448 factors (Bervan and Chadra 1988, Marshall et al. 2006), but what conditions are likely to

449 result in narrow fitness functions? Obviously constraints on maximum size will result in

450 narrowed fitness functions (Strathmann and Chaffee 1984, Congdon and Gibbons 1987,

451 Strathmann 1995). But there are factors other than functional constraints that penalize

452 offspring that are too large. For example, in the frog Bombina orientalis, tadpoles from larger

453 eggs can have lower performance than tadpoles from smaller eggs (Kaplan 1992). For

454 marine broadcast spawners, there is likely to be a narrow range of offspring sizes that results

455 in the optimal fertilization of eggs because fertilisation is egg size-dependent. Smaller eggs

456 face sperm limitation and larger eggs may suffer from polyspermy (Marshall et al. 2002). In

457 species with non-feeding larvae, egg size is positively correlated with development time and 
458 as such, larger eggs will remain in the plankton for longer (reviewed in Marshall and Keough

459 2007). Given that mortality rates in the plankton can be very high, increasing offspring size

460 in species with non-feeding larvae may reduce rather than increase offspring fitness. As a

461 result of such factors, only a narrow range of offspring sizes may be viable. In species such

462 as these, we suggest that even a small level of environmental unpredictability may result in

463 variable strategies being favored (for a more detailed review of the negative effects of

464 increasing offspring size see Bernardo 1996). We should note that we do not suggest that all

465 within brood variation in offspring size is necessarily adaptive, merely that there is an

466 adaptive element to this variation when environments are unpredictably variable and

467 production of offspring that are too large or too small results in direct fitness costs. Our

468 results are robust to a 16-fold difference in the viable egg size range. However, it would be

469 interesting to investigate the consequences of altering the shape of the egg fitness function in

470 a variety of other ways (e.g., disruptive selection on egg size).

471 The theoretical finding that within-brood variation in egg size can represent an

472 adaptive form of diversified bet-hedging in highly unpredictable environments accords with

473 comparative empirical evidence, which shows that within-brood variation in offspring size is

474 relatively low in species where mothers have greater opportunity to anticipate the

475 relationship between offspring size and performance (i.e., direct developers), but high in

476 species where this relationship is likely to be unpredictable (i.e., indirect developers with

477 feeding larvae). Based on the key assumption that offspring fitness is maximized at an

478 intermediate size, our theoretical analysis suggests that the observed relationship between

479 environmental predictability and within-brood variation in offspring size in marine

480 invertebrates reflects adaptive variation in reproductive strategies. The converse pattern was 
481 observed for variation among mothers. The high level of among-mother variation in

482 offspring size for direct developers is predicted to occur only if mothers can adjust the size of

483 their offspring according to local conditions. If mothers can produce offspring that are close

484 to the optimal size, then fitness will be higher under an invariant strategy. While adaptive

485 plasticity in offspring size has been demonstrated in insects (Fox et al. 1997), we know of no

486 study that has demonstrated this in marine direct developers.

487 Previous studies have examined variation in offspring size in more or less predictable

488 environments but they did not partition variation into among- and within-mother levels

489 (Poulin and Hamilton 2000, Einum and Fleming 2002, Dziminski and Alford 2005). Our

490 findings in both the simulations and the comparative analysis highlight the fact that selection

491 will act very differently on these two sources of variation, suggesting that they should be

492 considered separately. Environmental unpredictability should select for increased among-

493 mother variation for species that can predict the environment that their offspring will

494 encounter, whereas it should select for increased within-brood variation for species that

495 cannot predict the conditions that their offspring are likely to experience.

496 Both non-feeding and feeding indirect developers had much lower levels of among-

497 mother variation in offspring size than direct developers, but only the indirect developers

498 with feeding larvae had high levels of within-brood variation. This accords with our model's

499 predictions: most indirect developers with feeding larvae spend weeks to months in the

500 plankton, passing through multiple life-history stages, and disperse to habitats far removed

501 from the maternal habitat. The different developmental modes differed not only in their

502 dispersal but also the number of life-history stages at which offspring size could affect

503 performance. For example, fertilization was external in most of the indirect developers and 
504 other studies have shown that egg size can affect fertilization kinetics in external fertilizers

505 (Levitan 1996, Marshall et al. 2002). Thus, we would expect that the relationship between

506 offspring size and performance would be least predictable in this group and, accordingly,

507 high levels of offspring size variation within-broods should be favored. It should be noted

508 that the species within the different developmental modes vary across a range of different

509 life-history traits, all of which have the potential to affect offspring size variation. For

510 example, all of the direct developing species have a mobile adult stage but many of the

511 indirect developers were sessile as adults. Thus we cannot rule out other factors that may also

512 affect the differences in offspring size variation observed in this study.

513 An alternative explanation for the high levels of variation in offspring size among

514 mothers in direct developers is that maternal phenotype and the natal environment are linked.

515 Larger mothers typically produce more offspring and, in weakly dispersing species, this may

516 result in higher levels of sibling competition. McGinley et al. (1987) suggest that larger

517 mothers may therefore provision their offspring with more resources (i.e. make them larger)

518 to deal with the increased levels of sibling competition, thus producing a correlation between

519 maternal and offspring size. In many marine invertebrates, offspring size is correlated with

520 maternal size (Marshall et al. 2000, Marshall and Keough 2003) but it is, as yet, unclear

521 whether this relationship is more common in direct developers.

522 Overall, our theoretical and comparative analyses support the view that within-brood

523 variation in offspring size, like mean offspring size, is under selection. When environment is

524 unpredictably variable, and the range of viable offspring sizes is relatively narrow, selection

525 is likely to favor production of offspring of variable sizes within broods. In contrast, stable

526 environments and a large range of viable offspring sizes will generally favor a constant 
527 offspring size within broods. One component that theoretical considerations of offspring size,

528 including our own, fail to incorporate is the physiological cost of producing offspring of

529 uniform size. We agree with Fox and Czesak (2000)'s suggestion that at least some of the

530 within-brood variation in offspring size may reflect the cost associated with the production of

531 uniformly sized offspring, or physiological constraints against uniform offspring size.

532 Nevertheless, it is clear that systematic differences in the level of within-brood variation

533 occur among organisms with different life-history strategies and we suggest that these

534 differences may be adaptive.

535

\section{Acknowledgements}

537 We thank Maria Byrne for generously providing unpublished data on egg size variation in

538 sea-stars. We thank Troy Day, Pat Krug and Mick Keough for helpful discussions of the

539 topic which helped us clarify our thoughts on several issues. We also thank David Schiel and

540 two anonymous reviewers for thoughtful reviews that greatly improved the manuscript. DJM

541 and RB were supported by Australian Post-Doctoral Fellowships from the Australian

542 Research Council. LFB was supported by a post-doctoral fellowship from NSERC (Canada),

543 a University of New South Wales Faculty Research Grant to him and Robert Brooks, and the

544 University of Zürich.

\section{Literature Cited}

547 Bagenal, T. B. 1969. Relationship between egg size and fry survival in brown trout Salmo

548 trutta L. Journal of Fish Biology 1:349-353. 
Bernardo, J. 1996. The particular maternal effect of propagule size, especially egg size: 236.

Bervan, K. A., and B. G. Chadra. 1988. The relationship among egg size, density and food level on larval development in the wood frog (Rana sylvatica). Oecologia 75:67-72.

Bull, J. J. 1987. Evolution of phenotypic variance. Evolution 41:303-315.

Capinera, J. L. 1979. Qualitative variation in plants and insects - effect of propagule size on ecological plasticity. American Naturalist 114:350-361.

Cohen, D. 1966. Optimizing reproduction in a randomly varying environment. Journal of Theoretical Biology 12:119-129.

Collin, R. 2000. Sex change, reproduction, and development of Crepidula adunca and Crepidula lingulata (Gastropoda: Calyptraeidae). Veliger 43:24-33.

561 Congdon, J. D., and J. W. Gibbons. 1987. Morphological constraint on egg size - a challenge to optimal egg size theory. Proceedings of the National Academy of Sciences of the United States of America 84:4145-4147.

Cooper, W. S., and R. H. Kaplan. 1982. Adaptive "coin-flipping": A decision-theoretic examination of natural selection for random individual variation. Journal of Theoretical Biology 94:135-151.

Crump, M. L. 1981. Variation in propagule size as a function of environmental uncertainty

569 Dibattista, J. D., K. A. Feldheim, S. H. Gruber, and A. P. Hendry. 2007. When bigger is not 570 better: selection against large size, high condition and fast growth in juvenile lemon sharks. Journal of Evolutionary Biology 20:201-212. 
572 Dziminski, M. A., and R. A. Alford. 2005. Patterns and fitness consequences of intraclutch

573 variation in egg provisioning in tropical Australian frogs. Oecologia 146:98-109.

574 Einum, S., and I. A. Fleming. 2000. Highly fecund mothers sacrifice offspring survival to

$575 \quad$ maximize fitness. Nature 405:565-567.

576 Einum, S., and I. A. Fleming. 2002. Does within-population variation in fish egg size reflect

577 maternal influences on optimal values. American Naturalist 160:756-765.

578 Einum, S., and I. A. Fleming. 2004a. Does within-population variation in egg size reduce

579 intraspecific competition in Atlantic Salmon, Salmo salar? Functional Ecology

$580 \quad 18: 110-115$

581 Einum, S., and I. A. Fleming. 2004b. Environmental unpredictability and offspring size:

582 conservative versus diversified bet-hedging. Evolutionary Ecology Research 6:443-

$583 \quad 455$.

584 Emlet, R. B. 1986. Facultative planktotrophy in the tropical echinoid Clypaster rosaceus

585 (Linnaeus) and a comparison with obligate planktotrophy in Clypaster subdepressus

586 (Gray) (Clypasteroida: Echinoidea). Journal of Experimental Marine Biology

$587 \quad$ Ecology 95:183-202.

588 Fox, C. W., Thakar, M. S. and Mosseau, T. A. (1997). Egg size plasticity in a seed beetle: An

589 adaptive maternal effect. American Naturalist 149: 149-163.

590 Fox, C. W., and M. E. Czesak. 2000. Evolutionary ecology of progeny size in arthropods.

591 Annual Review of Entomology 45:341-369.

592 Geritz, S. A. H. 1995. Evolutionarily stable seed polymorphism and small-scale spatial

$593 \quad$ variation in seedling density. American Naturalist 146:685-707. 
594 Gillespie, J. H. 1977. Natural selection for variances in offspring numbers: a new

595 evolutionary principle. American Naturalist 111:1010-1014.

596 Hadfield, M. G., and M. F. Strathmann. 1996. Variability, flexibility and plasticity in life 597 histories of marine invertebrates. Oceanologica acta 19 323-334.

598 Hart, M. W., M. Byrne, and S. L. Johnson. 2003. Patiriella pseudoexigua (Asteroidea :

599 Asterinidae): a cryptic species complex revealed by molecular and embryological analyses. Journal of the Marine Biological Association of the United Kingdom

83:1109-1116.

Harvey, P. H., and M. D. Pagel. 1991, The comparative method in evolutionary biology, Oxford University Press.

Havenhand, J. N. 1995. Evolutionary ecology of larval types. Pages 79-122 in L. R. McEdward editor. Ecology of marine invertebrate larvae. CRC, Boca Raton.

Hendry, A. P., T. Day, and A. B. Cooper. 2001. Optimal size and number of propagules: Allowance for discrete stages and effects of maternal size on reproductive output and offspring fitness. American Naturalist 157:387-407.

Kaplan, R. H. 1992. Greater maternal investment can decrease offspring survival in the frog Bombina orientalis. Ecology 73:280-288.

611 Kohn, A. J., and F. E. Perron. 1994. Life-history and biogeography patterns in Conus. Clarendon Press, Oxford.

613 Krug, P. J. 1998. Poecilogony in an estuarine opisthobranch: planktotrophy, lecithotrophy, 614 and mixed clutches in a population of the ascoglossan Alderia modesta. Marine

615 Biology 132:483-494. 
616 Kudo, S. 2001. Intraclutch egg-size variation in acanthosomatid bugs: adaptive allocation of 617 maternal investment. Oikos 92:208-214.

618 Laaksonen, T. 2004. Hatching asynchrony as a bet-hedging strategy - an offspring diversity 619 hypothesis. Oikos 104:616-620.

620 Lack, D. 1947. The significance of clutch size. Ibis 89:302-352.

621 Levitan, D. R. 1993. The importance of sperm limitation to the evolution of egg size in 622 marine invertebrates. American Naturalist 141:517-536.

623 Levitan, D. R. 1996. Effects of gamete traits on fertilisation in the sea and the evolution of 624 sexual dimorphism. Nature 382: 153-155.

625 Levitan, D. R. 2000. Optimal egg size in marine invertebrates: theory and phylogenetic 626 analysis of the critical relationship between egg size and development time in echinoids. American Naturalist 156:175-192.

628 Lips, K. R. 2001. Reproductive trade-offs and bet-hedging in Hyla calypsa, a Neotropical treefrog. Oecologia 128:509-518.

630 Lloyde, D. G. 1987. Selection of offspring size at independence and other size-versusnumber strategies. American Naturalist 129:800-817.

632 Marshall, D. J., T. F. Bolton, and M. J. Keough. 2003. Offspring size affects the post633 metamorphic performance of a colonial marine invertebrate. Ecology 84:3131-3137.

634 Marshall, D. J., M. J. Keough 2007. Evolutionary ecology of offspring size in marine 635 invertebrates. Advances in Marine Biology 53: 1-60.

636 Marshall, D.J., C. N. Cook, and R. B. Emlet. 2006. Offspring size effects mediate 637 competition in a colonial marine invertebrate. Ecology 87: 214-225. 
638 Marshall, D. J., and M. J. Keough. 2003. Sources of variation in larval quality for free-

639 spawning marine invertebrates: Egg size and the local sperm environment.

640 Invertebrate Reproduction and Development 44:63-70.

641 Marshall, D. J., C. A. Styan, and M. J. Keough. 2000. Intraspecific co-variation between egg

642 and body size affects fertilisation kinetics of free-spawning marine invertebrates.

643 Marine Ecology Progress Series 195:305-309.

644 Marshall, D. J., C. A. Styan, and M. J. Keough. 2002. Sperm environment affects offspring

645 quality in broadcast spawning marine invertebrates. Ecology Letters 5:173-176.

646 McGinley, M. A., D. H. Temme, and M. A. Geber. 1987. Parental investment in offspring in

647 variable environments: theoretical and empirical considerations. American Naturalist

$648 \quad$ 130:370-398.

649 Moran, A. L., and R. B. Emlet. 2001. Offspring size and performance in variable

650 environments: field studies on a marine snail. Ecology 82:1597-1612.

651 Orr, H., A. 2007. Absolute fitness, relative fitness and utility. Evolution 61: 2997-3000.

652 Parker, G. A., and M. Begon. 1986. Optimal egg size and clutch size - effects of environment 653 and maternal phenotype. American Naturalist 128:573-592.

654 Podolsky, R., D., and R. Strathmann, R. 1996. Evolution of egg size in free spawners:

655 consequences of the fertilisation-fecundity trade-off. American Naturalist 148:160-

656173.

657 Podolsky, R. D. 2002. Fertilization ecology of egg coats: physical versus chemical

658 contributions to fertilization success of free-spawned eggs. Journal of Experimental

659 Biology 205:1657-1668. 
660 661

662

663

664

665

666

667

668

669

670

671

672

673

674

675

676

677

678

679

680

681

Poulin, R., and W. J. Hamilton. 2000. Egg size variation as a function of environmental variability in parasitic trematodes. Canadian Journal of Zoology-Revue Canadienne De Zoologie 78:564-569.

Roff, D. A. 1992. The evolution of life histories. Chapman \& Hall, New York.

Sakai, S., and Y. Harada. 2001. Why do large mothers produce large offspring? Theory and a test. American Naturalist 157:348-359.

Sargent, R. C., P. D. Taylor, and M. R. Gross. 1987. Parental care and the evolution of egg size in fishes. American Naturalist 129:32-46.

Seger, J., and H. J. Brockman. 1987. What is bet-hedging? Oxford Surveys in Evolutionary Biology 182-211.

Simons, A. M., and M. O. Johnston. 1997. Developmental instability as a bet-hedging strategy. Oikos 80:401-406.

Simons, A. M., and M. O. Johnston. 2006. Environmental and genetic sources of diversification in the timing of seed germination: Implications for the evolution of bet hedging. Evolution 60:2280-2292.

Smith, C. C., and S. D. Fretwell. 1974. The optimal balance between size and number of offspring. American Naturalist 108:499-506.

Stearns, S. C. 1992. The evolution of life histories. Oxford University Press, Oxford.

Strathmann, R. R. 1995. Peculiar constraints on life histories imposed by protective or nutritive devices for embryos. American Zoologist 35:426-433.

Strathmann, R. R., and C. Chaffee. 1984. Constraints on egg masses. II. Effect of spacing, size and number of eggs on ventiliation of masses of embryos in jelly, adherent 

84:85-93.

684 Styan, C. A. 1998. Polyspermy, egg size and the fertilization kinetics of free-spawning marine invertebrates. American Naturalist 152:290-297.

686 Turner, R., and J. M. Lawrence. 1977. Volume and composition of echinoderm eggs:

687 implications for the use of egg size in life-history models, Pages $25-40$ in S. E.

688 Stancyk, ed., Reproductive ecology of marine invertebrates. Columbia, University of

$689 \quad$ South Carolina Press

690 Vance, R., R. 1973. On reproductive strategies in marine benthic invertebrates. American Naturalist 107:339-352.

692 Williams, M. D. 1994. Intraspecific variation in egg size and egg composition in birds: effects on offspring fitness. Biological Review 68:38-59. 